\title{
Natural convection in a vertical slot: accurate solution of the linear stability equations
}

\author{
$\begin{array}{ll}\text { G. D. McBain* } & \text { S. W. Armfield }\end{array}$
}

(Received 8 August 2003; revised 12 March 2004)

\begin{abstract}
The linear stability of natural convection in a fluid between vertical hot and cold walls was studied using a collocation method. Seven figure accurate results for monotonic disturbances were obtained by Ruth (1979) using numerical power series, but this method is intrinsically limited and failed for $\operatorname{Pr}>10$. In contrast, Chebyshev collocation converges more rapidly and allows the computation of results at higher Pr for which oscillatory disturbances dominate. Accurate results are now obtained across the entire Prandtl number range. These match the zero and infinite Pr asymptotes which are also refined here.
\end{abstract}

${ }^{*}$ School of Aerospace, Mechanical \& Mechatronic Engineering, The University of Sydney, Australia. mailto:geordie.mcbain@aeromech.usyd.edu.au

†ditto, mailto: armfield@aeromech.usyd.edu.au

See http://anziamj.austms.org.au/V45/CTAC2003/McB2/home.html for this article, (C) Austral. Mathematical Soc. 2004. Published March 23, 2004. ISSN 1446-8735 


\section{Contents}

1 Introduction

C93

2 Problem formulation

C95

2.1 Governing equations . . . . . . . . . . . . C95

2.2 Zero Prandtl number . . . . . . . . . . . . . . . . C C96

2.3 Large Prandtl number . . . . . . . . . . . . . . . C96

3 Numerics

C98

3.1 Discretization . . . . . . . . . . . . . . . . C98

3.2 Solution of the algebraic problems . . . . . . . . . . C99

3.3 Organization of the computations . . . . . . . . . C99

4 Results

4.1 Stability margins . . . . . . . . . . . . . . C100

4.2 Critical Grashof numbers . . . . . . . . . . . . . C101

5 Conclusion

C103

References

C103

\section{Introduction}

Natural convection between vertical walls held at different temperatures is a classical problem in fluid mechanics [13] and has applications in the separation of isotopes [11] and the insulation value of double-pane windows [2]. Laminar convection (Figure 1) is unstable if the temperature difference (or, in dimensionless terms, the Grashof number, Gr) is sufficiently high. Here we seek the critical Gr: the smallest Gr for which the flow becomes unstable to infinitesimal perturbations. 


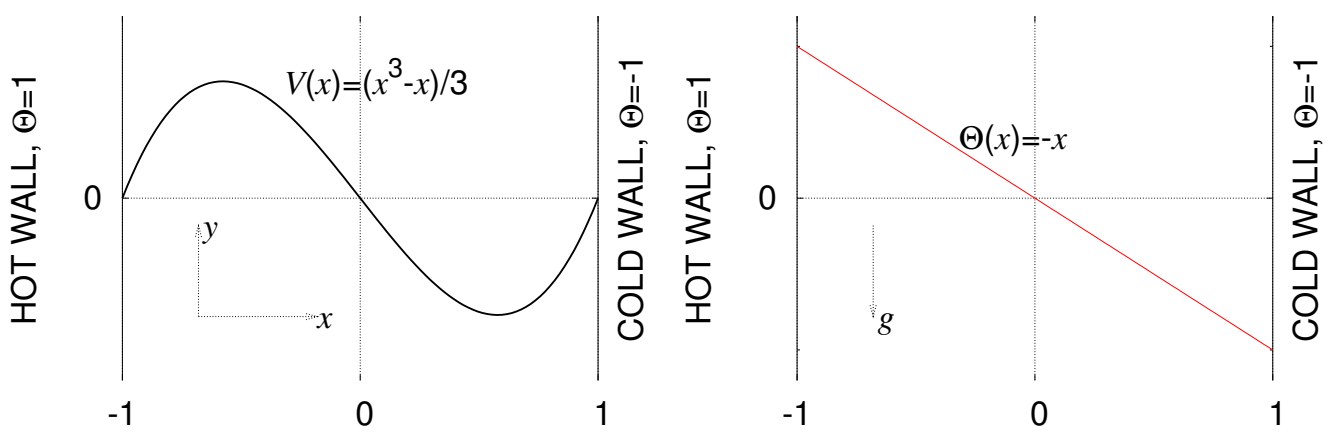

Figure 1: Laminar vertical slot natural convection [20].

Most previous numerical methods, such as eigenfunction expansions [12, 6] and numerical power series [17], failed for large Prandtl numbers (Pr: the ratio of kinematic viscosity to thermal diffusivity). In another problem where oscillatory disturbances are important - convection in fluids with variable properties - Suslov \& Paolucci [19] achieved high accuracy with a Chebyshev collocation method. Adopting a similar method here, we have extended the accurate results of Ruth [17] for $\operatorname{Pr} \leqslant 10$ across the entire Pr number range. Further, the method is applied to the $\operatorname{Pr} \rightarrow 0$ [4] and $\operatorname{Pr} \rightarrow \infty$ [10] equations to find the asymptotes to the same high precision.

The high Pr behaviour turns out to be quite complicated, with the final asymptotic behaviour not appearing until $\operatorname{Pr}$ is higher than $10^{3}$; this may be relevant to flows of $\operatorname{Pr} \approx 10^{4}-10^{6}$ liquids like lava or cane molasses. 


\section{Problem formulation}

\subsection{Governing equations}

The dimensionless Oberbeck equations of natural convection are [15]

$$
\begin{aligned}
\left(\frac{\partial}{\partial t}+\mathbf{u} \cdot \nabla-\frac{32}{\mathrm{Gr}} \nabla^{2}\right) \mathbf{u} & =-\nabla p+\frac{64}{\mathrm{Gr}} T \hat{\boldsymbol{\jmath}}, \\
\left(\operatorname{Pr}\left\{\frac{\partial}{\partial t}+\mathbf{u} \cdot \nabla\right\}-\frac{32}{\mathrm{Gr}} \nabla^{2}\right) T & =0,
\end{aligned}
$$

and $\nabla \cdot \mathbf{u}=0$. Steady solutions with zero net vertical flow rate and independent of altitude $y$ satisfy $\mathbf{u}=V(x) \hat{\boldsymbol{\jmath}}$ and $T=\Theta(x)$, where $V^{\prime \prime}(x)=-2 \Theta(x)$ and $\Theta^{\prime \prime}(x)=0$. For boundary conditions $V( \pm 1)=0, \Theta( \pm 1)=\mp 1$, the exact solution is $V(x)=\left(x^{3}-x\right) / 3$ and $\Theta(x)=-x$ [20]; see Figure 1 .

The evolution of infinitesimal plane disturbances is governed by the OrrSommerfeld equation with a buoyancy term, plus a similar equation for the temperature fluctuation [8]. In block form $L q=c M q$, where $L=$

$$
\begin{gathered}
{\left[\begin{array}{cc}
\frac{\mathrm{i} \alpha \mathrm{Gr}}{64}\left\{V\left(\frac{\alpha^{2}}{4}-\mathrm{D}^{2}\right)+V^{\prime \prime}\right\}+\left(\frac{\alpha^{2}}{4}-\mathrm{D}^{2}\right)^{2} & 2 \mathrm{D} \\
-\Theta^{\prime} & V+\frac{64}{\mathrm{i} \alpha \mathrm{GrPr}}\left(\frac{\alpha^{2}}{4}-\mathrm{D}^{2}\right)
\end{array}\right],} \\
M=\left[\begin{array}{cc}
\frac{\mathrm{i} \alpha \mathrm{Gr}}{4}\left(\frac{\alpha^{2}}{4}-\mathrm{D}^{2}\right) & 0 \\
0 & 16
\end{array}\right], \quad q=\left[\begin{array}{c}
\psi \\
\theta
\end{array}\right],
\end{gathered}
$$

and $D \equiv \mathrm{d} / \mathrm{d} x$. The boundary conditions are $\psi( \pm 1)=\psi^{\prime}( \pm 1)=0$ and $\theta( \pm 1)=0$. This is an eigenvalue problem for the complex wave speed $c$. Modes are stable or unstable as $\Im c<0$ or $\Im c>0$. 


\subsection{Zero Prandtl number}

As $\operatorname{Pr} \rightarrow 0$ with $\mathrm{Gr}=\mathcal{O}(1)$, the temperature perturbation equation reduces to $\left(\alpha^{2} / 4-\mathrm{D}^{2}\right) \theta=0$, so that all temperature perturbations decay [9], and the stability problem reduces to an Orr-Sommerfeld equation [4]

$$
\left[\frac{\mathrm{i} \alpha \mathrm{Gr}}{64}\left\{V\left(\frac{\alpha^{2}}{4}-\mathrm{D}^{2}\right)+V^{\prime \prime}\right\}+\left(\frac{\alpha^{2}}{4}-\mathrm{D}^{2}\right)^{2}\right] \psi=c \frac{\mathrm{i} \alpha \mathrm{Gr}}{4}\left(\frac{\alpha^{2}}{4}-\mathrm{D}^{2}\right) \psi
$$

\subsection{Large Prandtl number}

Results at high Pr for a related problem guided Gill \& Kirkham [10] to the appropriate asymptotic expansion: powers of $\mathrm{iPr}^{-1 / 2}$ with $\mathrm{Gr}=\mathcal{O}\left(\mathrm{Pr}^{-1 / 2}\right)$. Explicitly, set $S=\operatorname{GrPr}^{1 / 2}, q=q_{0}+\mathrm{iPr}^{-1 / 2} q_{1}, c=c_{0}+\mathrm{iPr}^{-1 / 2} c_{1}, L=$ $L_{0}+\mathrm{iPr}^{-1 / 2} L_{1}$, and $M=M_{0}+\mathrm{iPr}^{-1 / 2} M_{1}$. Then

$$
\begin{aligned}
(L-c M) q \sim & \left(L_{0}-c_{0} M_{0}\right) q_{0} \\
& +\left[\left(L_{0}-c_{0} M_{0}\right) q_{1}-\left\{c_{1} M_{0}-\left(L_{1}-c_{0} M_{1}\right)\right\} q_{0}\right] \operatorname{Pr}^{-1 / 2} \\
& +\mathcal{O}\left(\operatorname{Pr}^{-1}\right), \quad(\operatorname{Pr} \rightarrow \infty) .
\end{aligned}
$$

Here, at zeroth order, there is a generalized eigenvalue problem

$$
\left(L_{0}-c_{0} M_{0}\right) q_{0}=0
$$

and at first order an inhomogeneous equation

$$
\left(L_{0}-c_{0} M_{0}\right) q_{1}=\left\{c_{1} M_{0}-\left(L_{1}-c_{0} M_{1}\right)\right\} q_{0} .
$$

Let $\langle\cdot, \cdot\rangle$ denote some inner product and $A^{*}$ the adjoint of an operator $A$ so that $\langle A q, r\rangle=\left\langle q, A^{*} r\right\rangle$ for all $q$ and $r$. Assume $r$ is a solution of the adjoint of $(7),\left(L_{0}-c_{0} M_{0}\right)^{*} r=0$. The inner product of an arbitrary $\chi$ with either side 
of this equation gives $\left\langle\chi,\left(L_{0}-c_{0} M_{0}\right)^{*} r\right\rangle=0$, so that $\left\langle\left(L_{0}-c_{0} M_{0}\right) \chi, r\right\rangle=0$. Putting $q_{1}$ from (8) for $\chi$, we obtain an equation for the determination of $c_{1}$ :

$$
c_{1}\left\langle M_{0} q_{0}, r\right\rangle=\left\langle\left(L_{1}-c_{0} M_{1}\right) q_{0}, r\right\rangle .
$$

The point of this abstract discussion is that any inner product can be used. Gill \& Kirkham [10] used $\left\langle q_{1}, q_{2}\right\rangle=\int_{-1}^{1}\left(\psi_{2}^{*} \psi_{1}+\theta_{2}^{*} \theta_{1}\right) \mathrm{d} x$. However, after discretization $\mathbf{q}$ is a vector and an alternative is the Euclidean scalar product $\langle q, r\rangle=\mathbf{q} \cdot \mathbf{r}$. This is both more convenient and more accurate numerically.

Since we are more interested in $S$ than $c_{1}$ at the neutral condition

$$
\Im c=0,
$$

we decompose $L_{1}=S L_{11}+L_{12} / S$ and $M_{1}=S M_{11}$ in (9) so that (10) with $c \sim c_{0}+\mathrm{iPr}^{-1 / 2} c_{1}$ becomes $\operatorname{Pr}^{-1 / 2} \Re c_{1}=-\Im c_{0}$ or

$$
S^{2} \Re\left\{\kappa\left\langle\left(L_{11}-c_{0} M_{11}\right) q_{0}, r\right\rangle\right\}+S \operatorname{Pr}^{1 / 2} \Im c_{0}+\Re\left\{\kappa\left\langle L_{12} q_{0}, r\right\rangle\right\}=0,
$$

where $\kappa=\left\langle M_{0} q_{0}, r\right\rangle^{-1}$.

The operators appearing in this quadratic for $S$ are

$$
L_{11}-c_{0} M_{11}=\left[\begin{array}{cc}
\frac{\alpha}{64}\left\{\left(V-16 c_{0}\right)\left(\frac{\alpha^{2}}{4}-D^{2}\right)+V^{\prime \prime}\right\} & 0 \\
0 & 0
\end{array}\right],
$$

and

$$
L_{12}=\left[\begin{array}{cc}
0 & 0 \\
0 & -\frac{64}{\alpha}\left(\frac{\alpha^{2}}{4}-D^{2}\right)
\end{array}\right] \text {. }
$$

It turns out that for the problems of interest the zeroth order eigenvalues and eigenvectors are real; in this case we have

$$
S_{\text {crit }}=\left\{\frac{-\left\langle L_{12} q_{0}, r\right\rangle}{\left\langle\left(L_{11}-c_{0} M_{11}\right) q_{0}, r\right\rangle}\right\}^{1 / 2} .
$$




\section{Numerics}

\subsection{Discretization}

The above problems were discretized by ordinate-based interior collocation. That is, if $\left\{x_{i}\right\}_{1}^{n}$ are distinct points in $[-1,1]$ and $\left\{\phi_{j}(x)\right\}_{1}^{n}$ polynomials of degree $n-1$ with the property $\phi_{j}\left(x_{i}\right)=\delta_{i j}$, so that for any function $f(x)$, $\sum_{j} \phi_{j}(x) f\left(x_{j}\right)$ is the polynomial interpolant, then differentiation is approximated by differentiation of these interpolants. The $d$ th derivative at the $i$ th point can be written as a matrix-vector product:

$$
\sum_{j} \phi_{j}^{(d)}\left(x_{i}\right) f\left(x_{j}\right) \equiv \sum_{j} D_{i j}^{(d)} f_{j}
$$

Boundary conditions are enforced by multiplying each basis function by an appropriate coercion function: $\tilde{\phi}_{j}(x) \equiv \beta_{j}(x) \phi_{j}(x) / \beta_{j}\left(x_{j}\right)$. These retain the interpolant property $\tilde{\phi}_{j}\left(x_{i}\right)=\delta_{i j}$. For example, $\beta_{j}(x)=\left(1-x^{2}\right)^{p}$ forces $f^{(q)}( \pm 1)=0$ for $q<p$. The modified differentiation matrices are given by

$$
\tilde{D}_{i j}^{(d)}=\sum_{k=0}^{d}\left(\begin{array}{l}
d \\
k
\end{array}\right) \frac{\beta_{j}^{(d-k)}\left(x_{i}\right)}{\beta_{j}\left(x_{j}\right)} D_{i j}^{(k)} .
$$

Here the Chebyshev-Lobatto points

$$
\left\{x_{i}\right\}_{1}^{n}=\cos \frac{j \pi}{n+1}
$$

are used so that

$$
\phi_{j}(x)=\frac{T_{n+1}^{\prime}(x)}{\left(x-x_{j}\right) T_{n+1}^{\prime \prime}\left(x_{j}\right)},
$$

where $T_{n}(\cos \theta)=\cos (n \theta)$. 
For the second derivative matrix, $\mathbf{D}^{(2)}$, the coercion function is $\beta(x)=$ $\left(1-x^{2}\right)$, and $\beta(x)=\left(1-x^{2}\right)^{2}$ for $\mathbf{D}^{(4)}$. The use of different coercion functions and therefore interpolants for the same function may seem unnatural, but is necessary to prevent the 'mass matrix' $\mathbf{M}$ in (4) from being overdetermined which would introduce spurious eigenvalues [21].

The computations were carried out in Octave [7] using a public domain collocation library [21].

\subsection{Solution of the algebraic problems}

The discrete generalized eigenvalue problems $(\mathbf{L}-c \mathbf{M}) \mathbf{q}=0$ were converted to standard form in two ways.

First, since $\mathbf{M}$ is invertible due to the careful imposition of boundary conditions (see § 3.1), an equivalent problem is $\mathbf{M}^{-1} \mathbf{L q}=c \mathbf{q}$. The discrete spectrum was then obtained from the $Q R$ Schur factorization, as implemented in LAPACK's ZGEEV [1] and Octave's eig. This transformation is unavailable for the high Pr problem (7) since $M_{0}=\left[\begin{array}{ll}0 & 0 \\ 0 & 16\end{array}\right]$ is singular.

Second, if $\sigma$ is not an eigenvalue, $(\mathbf{L}-\sigma \mathbf{M})$ is invertible, and the shiftand-invert transformation [18] is $\mathbf{E} x=\mu x$ where $\mathbf{E}=(\mathbf{L}-\sigma \mathbf{M})^{-1} \mathbf{M}$ and $c=\sigma+1 / \mu$. The transformed problem was solved by simple power iteration. This method was directly coded in Octave. Better results were obtained after a two-sided diagonal scaling [1] was applied to $\mathbf{L}$ and $\mathbf{M}$.

\subsection{Organization of the computations}

The methods of $\S \S 3.1-3.2$ were used to solve problems at a given $\mathrm{Pr}, \mathrm{Gr}$, and $\alpha$. For given $\operatorname{Pr}$, the values of $\mathrm{Gr}$ and $\alpha$ were selected automatically by a new curve following method [14] so as to trace the linear stability margin 

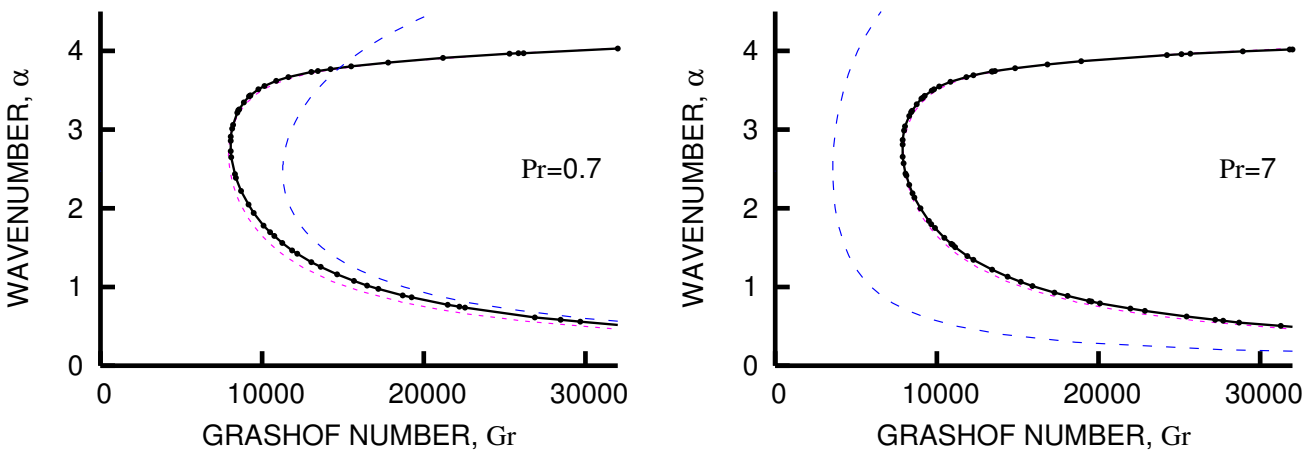

Figure 2: Linear stability margins at low Prandtl numbers $(\operatorname{Pr}=0.7$ and 7$)$, also showing (short dashes) $\operatorname{Pr} \rightarrow 0$ and (long dashes) $\operatorname{Pr} \rightarrow \infty$ asymptotes.

$\max \Im c=0$. Finally, critical values (minima of Gr with respect to $\alpha$ ) were refined by golden section search.

\section{Results}

\subsection{Stability margins}

The stability margins for the most important cases, air $(\operatorname{Pr} \approx 0.7)$ and water $(\operatorname{Pr} \approx 7)$, are shown in Figure 2; they differ little from the zero Pr asymptote.

As Pr increases, a second lobe representing oscillatory instability appears for a narrow range of $\alpha$ near $\mathrm{Gr} \sim 5700 / \alpha$ (Figure 3). The least $\operatorname{Pr}$ for which this occurs was first estimated at 11.4 [5], but this is a difficult problem. We found that for $\operatorname{Pr} \leqslant 11.57$ the lobe lies in $\{\alpha<0.1\} \cup\left\{\mathrm{Gr}>5 \times 10^{4}\right\}$.

As Pr increases further, the oscillatory lobe widens and for $\operatorname{Pr} \geqslant 12.454$ becomes dominant (Figure 3). This refines the estimates $\operatorname{Pr} \approx 12$ [5], 12.5 [6], 

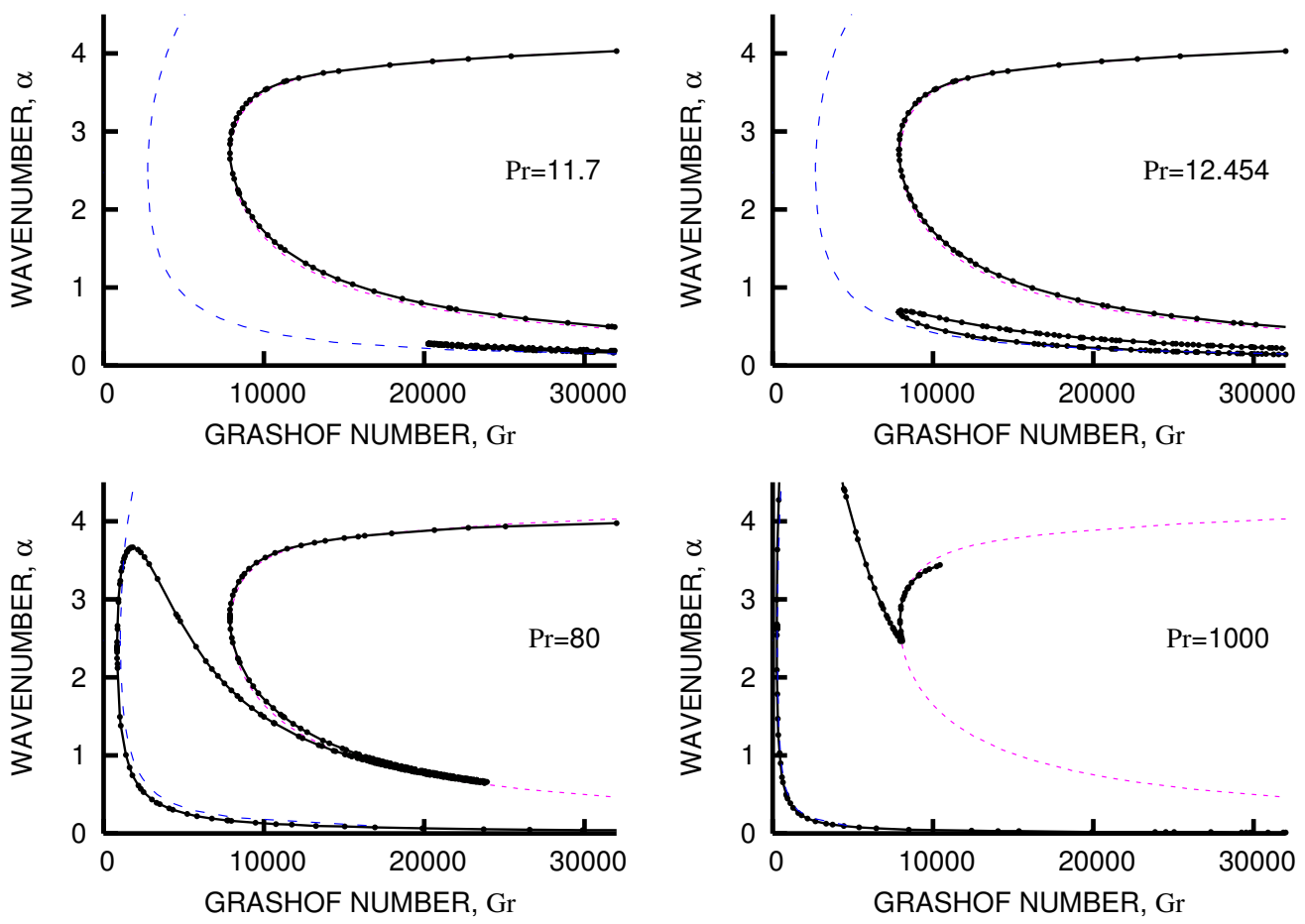

FiguRE 3: Linear stability margins at high Pr; see also Figure 2.

and corrects the widely quoted [3, 17, e.g.] 12.7 [12].

The two lobes merge, forming a cusp, somewhere in $20<\operatorname{Pr}<100$ [6]; this is another difficult number to estimate, but Figure 3 suggests it is near 80. The oscillatory lobe continues to widen as the Prandtl number increases. Its lower branch lies near the $\operatorname{Pr} \rightarrow \infty$ asymptote for all Pr.

\subsection{Critical Grashof numbers}

The critical parameters for linear instability are shown in Table 1 and Fig- 


\begin{tabular}{cccc||cccc} 
Pr & Gr & $\alpha$ & $c \times 10^{3}$ & Pr & Gr & $\alpha$ & $c \times 10^{3}$ \\
\hline 0 & 7930.0551 & 2.6883 & 0 & $10^{4}$ & 87.6102 & 2.5633 & \pm 8.470 \\
$10^{1}$ & 7871.4561 & 2.7665 & 0 & $10^{5}$ & 29.2301 & 2.5050 & \pm 8.491 \\
$10^{2}$ & 749.6258 & 2.4203 & \pm 8.168 & $10^{6}$ & 9.402155 & 2.4800 & \pm 8.497 \\
$10^{3}$ & 251.1985 & 2.6209 & \pm 8.403 & $\infty$ & $9435.4 / \sqrt{\operatorname{Pr}}$ & 2.4737 & \pm 8.494
\end{tabular}

TABle 1: Critical data, complementing Table 1 of Ruth [17].
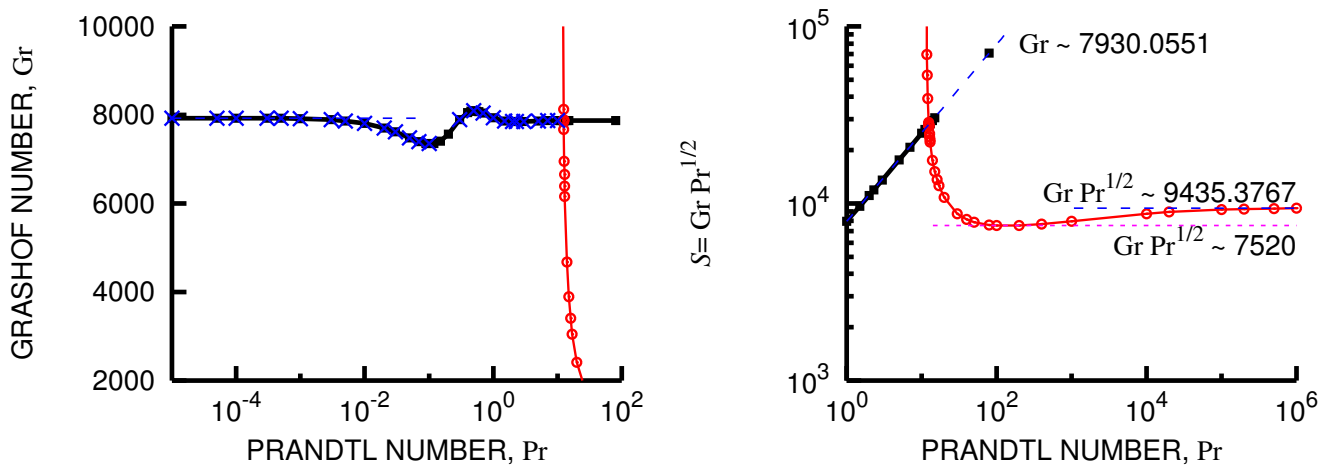

Figure 4: Critical Gr: monotonic (.) and oscillatory (o) modes, Ruth's data $(\times)$, the $\operatorname{Pr} \rightarrow 0$ and $\infty$ asymptotes, and a false asymptote [5].

ure 4. The dependence of the critical Grashof number on Pr for low Prandtl numbers is weak but complicated with a minimum near $\operatorname{Pr}=0.1$ and a maximum near 0.5 [16]. The monotonic branch lies near the $\operatorname{Pr} \rightarrow 0$ asymptote $(\mathrm{Gr} \sim 7930.0551)$ for all $\mathrm{Pr}$. Accurate critical data for $\operatorname{Pr}<10$ have been published by Ruth [17]. These were confirmed by the present study (Figure 4).

As noted in $\S 4.1$, the oscillatory mode becomes critical for $\operatorname{Pr}>12.454$. Hitherto [10] it was known that critical Gr $\sim$ const $\times \operatorname{Pr}^{-1 / 2}$, but two different values of the constant have been circulated: $9.4 \times 10^{3}$ [10] and $7.52 \times 10^{3}[5]$. The cause for this $20 \%$ discrepancy is now clear: the higher figure is based on a high Pr expansion (and has been here refined using the method of $\S 2.3$ 
to 9435.3767$)$; whereas the lower figure was apparently extrapolated from numerical results for Pr up to 100. It turns out that the lower 'asymptote' is just a stationary point and that above $\operatorname{Pr}=10^{2}$, the critical $\mathrm{GrPr}^{1 / 2}$ increases again, finally approaching the true asymptote for $\operatorname{Pr}>10^{5}$. Earlier large Prandtl numbers studies [12, e.g.] were unable to achieve sufficient accuracy to distinguish between these asymptotes or resolve this matter.

\section{Conclusion}

Chebyshev collocation allows accurate solution of the linear stability equations for natural convection in a vertical slot over the entire range of $\mathrm{Pr}$.

\section{References}

[1] E. Anderson, Z. Bai, C. Bischof, S. Blackford, J. Demmel, J. Dongarra, J. Du Croz, A. Greenbaum, S. Hammarling, A. McKenney, and D. Sorenson. LAPACK Users' Guide. SIAM, 3rd edition, 1999. C99

[2] G. K. Batchelor. Heat transfer by free convection across a closed cavity between vertical boundaries at different temperatures. Q. Appl. Math., 12:209-233, 1954. C93

[3] R. F. Bergholz. Instabilities of steady natural convection in a vertical fluid layer. J. Fluid Mech., 84:743-768, 1978. C101

[4] R. V. Birikh. On small perturbations of a plane parallel flow with cubic velocity profile. J. Appl. Math. Mech., 30:432-438, 1966. C94, C96

[5] R. V. Birikh, G. Z. Gershuni, E. M. Zhukhovitskii, and R. N. Rudakov. On oscillatory instability of plane-parallel convective motion in a vertical channel. J. Appl. Math. Mech., 36:707-710, 1972. C100, C102 
[6] Y.-M. Chen and A. J. Perlstein. Stability of free-convection flows of variable-viscosity fluids in vertical and inclined slots. J. Fluid Mech., 198:513-541, 1989. C94, C100, C101

[7] J. W. Eaton. GNU Octave Manual. Network Theory, 2002. C99

[8] G. Z. Gershuni. Ob ustoichivosti ploskogo konvectivnogo dvizheniia zhidkosti. Zhurnal Tekhnischeskoi Fiziki, 23:1838-1844, 1953. C95

[9] G. Z. Gershuni, E. M. Zhukhovitskii, and R. N. Rudakov. Heat perturbation spectrum of incompressible fluid flows. J. Appl. Math. Mech., 31:601-604, 1967. C96

[10] A. E. Gill and C. C. Kirkham. A note on the stability of convection in a vertical slot. J. Fluid Mech., 42:125-127, 1970. C94, C96, C97, C102

[11] R. C. Jones and W. H. Furry. The separation of isotopes by thermal diffusion. Rev. Mod. Phys., 18:151-224, 1946. C93

[12] S. A. Korpela, D. Gözüm, and C. B. Baxi. On the stability of the conduction regime of natural convection in a vertical slot. Intl J. Heat Mass Transfer, 16:1683-1690, 1973. C94, C101, C103

[13] G. D. McBain. Fully developed laminar buoyant flow in vertical cavities and ducts of bounded section. J. Fluid Mech., 401:365-377, 1999. C93

[14] G. D. McBain. Skirting subsets of the plane, with application to marginal stability curves. In Jagoda Crawford and A. J. Roberts, editors, Proc. of 11th Computational Techniques and Applications Conference CTAC-2003, volume 45, pages C78-C91, March 2004. [Online]

http://anziamj . austms . org. au/V45/CTAC2003/McBa/home.html [March 23, 2004]. C99 
[15] A. Oberbeck. Ueber die Wärmeleitung der Flüssigkeiten bei Berücksichtigung der Strömungen infolge von Temperaturdifferenzen. Ann. Phys. Chem., 7:271-292, 1879. Neue Folge. C95

[16] R. N. Rudakov. Spectrum of perturbations and stability of convective motion between vertical planes. J. Appl. Math. Mech., 31:376-383, 1967. C102

[17] D. W. Ruth. On the transition to transverse rolls in an infinite vertical fluid layer - a power series solution. Intl J. Heat Mass Transfer, 22:1199-1208, 1979. C94, C101, C102

[18] Y. Saad. Numerical Methods for Large Eigenvalue Problems. Manchester University Press, 1992. C99

[19] S. A. Suslov and S. Paolucci. Stability of natural convection flow in a tall vertical enclosure under non-Boussinesq conditions. Intl J. Heat Mass Transfer, 38:2143-2157, 1995. C94

[20] L. Waldmann. Zur Theorie des Gastrennungsverfahrens von Clusius und Dickel. Die Naturwissenschaften, 27:230-231, 1938. C94, C95

[21] J. A. C. Weideman and S. C. Reddy. A Matlab differentiation matrix suite. ACM Trans. Math. Software, 26:465-519, 2000. C99 